\title{
Clinical effect of platelet-rich fibrin on the preservation of the alveolar ridge following tooth extraction
}

\author{
YINGDI ZHANG, ZHENG RUAN, MINHUA SHEN, LUANJUN TAN, \\ WEIQIN HUANG, LEI WANG and YUANLIANG HUANG
}

Department of Stomatology, East Hospital Affiliated to Tongji University, Shanghai 200120, P.R. China

Received July 6, 2017; Accepted October 13, 2017

DOI: $10.3892 /$ etm.2018.5696

\begin{abstract}
The aim of the present study was to evaluate the clinical efficacy of platelet-rich fibrin (PRF) in preserving the alveolar ridge following human tooth extraction. A total of 28 patients were divided into two groups: The experimental and control groups ( $\mathrm{n}=14$ each). Following tooth extraction, the experimental group was implanted with PRF membrane, whereas the control group was not. The gingival healing effect was assessed at 7 days, 1 and 3 months later. Cone-beam computed tomography was performed immediately and at 3 months following tooth extraction. The changes in alveolar ridge height, width, and bone mineral density were compared between the two groups. The alveolar bone was removed using the ring drill during the implant surgery at 3 months following tooth extraction. Histomorphometric evaluation was performed to compare new bone formation between groups. The patients in the experimental group reportedly felt better compared with the patients in the control group. The healing of gingival tissue was better in the experimental group than in the control group. A significantly greater novel bone area was observed in the PRF group compared with the control group $(\mathrm{P}<0.01)$. However, no statistically significant differences were observed in the mean value of buccal alveolar ridge height, lingual/palatal alveolar ridge height and alveolar ridge width between the two groups. These results suggested that PRF was advantageous in human alveolar ridge preservation with ease of use and simple handling. Histological analysis of novel bone formation confirmed that PRF increased the quality of the novel bone and enhanced the rate of bone formation, despite the effect of PRF was not significant to reduce alveolar bone resorption in the extraction socket alone.
\end{abstract}

Correspondence to: Dr Lei Wang or Dr Yuanliang Huang, Department of Stomatology, East Hospital Affiliated to Tongji University, 150 Jimo Road, Shanghai 200120, P.R. China

E-mail: leiwang0222@163.com

E-mail: 13301921076@163.com

Key words: alveolar ridge preservation, cone-beam computing tomography, dental implant, platelet-rich fibrin

\section{Introduction}

In clinical practice, tooth loss is often caused by trauma, infection and periodontal disease amongst other reasons. The lack of stimulation of the surrounding ligament may lead to physiological bone resorption and, as a result, alveolar ridge height and width may be significantly reduced (1). In the physiological process of alveolar bone reconstruction, the bone width has been demonstrated to be decreased by $2.6-4.6 \mathrm{~mm}$, and the bone height reduced by 0.4-3.9 mm (2). According to a previous animal study, the absorption of labial alveolar bone was greater than that on the lingual side (3). In addition, the loss of alveolar bone is extremely unfavorable for dental restoration. An adequate quantity of bone is required as a basis for repair, not only for the fixed denture but also for the recoverable denture. Alveolar ridge preservation technology came into being to save more bone following tooth removal (4). Furthermore, it has previously been confirmed that alveolar ridge preservation technology may slow the alveolar bone resorption process and reduce the absorption of alveolar bone height and width (5).

Presently, the typically used method for alveolar ridge preservation is to fill the alveolar fossa $(6,7)$. The goal of the present study was to maintain the alveolar ridge height, width, and shape through the induction of osteogenesis. Previous research has been conducted on alveolar fossa materials such as granular or powdery hydroxyapatite (8), sponge-like polyethyl ester/polypropylene (9), bioactive glass (10) and injectable calcium phosphate (11), amongst others. Most of these biological materials are derived from heterogeneous organisms, resulting in the increased risk of tooth socket infection and economic burden for patients. It has also been demonstrated that many graft materials have not been completely degraded many years following implantation, and only slightly promote osteogenic induction (5), which also directly affects the formation of new bone and soft tissue healing in the tooth extraction sockets. Calcium phosphate cement and a variety of growth factors, including recombinant human bone morphogenetic protein-2 (rhBMP-2) and basic fibroblast growth factor (bFGF), have also been adopted to promote bone formation (12-14). Although these growth factors showed certain osteogenic ability, their relatively high price increased the cost of this potential treatment (14). Finding a more safe and inexpensive biological material for alveolar ridge preservation is a common goal in current research. 
Platelet-rich fibrin (PRF) is the second generation of platelet concentrates. Compared with the first generation of platelet-rich plasma (PRP), the preparation process is simple and does not require any thrombin or coagulant (15). PRF is harvested from venous blood and does not lead to immune rejection in clinical application $(15,16)$.

PRF has a three-dimensional network structure with is both flexible and durable (16). PRF is rich in fibrin, platelets, white blood cells, growth factors, cytokines, and other components conducive to tissue repair (16-18). These cytokines include interleukin-1, -4 and -6 , and other growth factors including platelet-derived growth factor, epithelial growth factor and vascular endothelial growth factor $(17,18)$. These components can be effective in regulating the proliferation, differentiation and apoptosis of repair-related cells, and subsequently regulating and promoting tissue repair (19). Leukocytes are able to release a large number of immune regulation-related cytokines in the process of fibrinolysis, and this process is persistent and progressive $(20,21)$. These cytokines may effectively reduce local inflammatory responses effectively (22). The fibrous web in the PRF, along with the large number of platelets and growth factors, may also promote wound healing and accelerate bone regeneration (23). In view of the aforementioned organizational characteristics and biological characteristics, PRF is safe, effective and more economical as a transplant material for alveolar site preservation.

A number of previous literature reports have confirmed that PRF may be used for alveolar ridge preservation and is beneficial to clinical application (24-30). Previous animal experiments have confirmed that, whether used in conjunction with Bio-Oss or used alone in animal bone defect models, PRF has exhibited good osteogenic ability and was able to alleviative soft tissue inflammation $(24,25)$. PRF has also been used for clinical alveolar site preservation and alveolar bone defect repair, which confirmed that it was able to promote bone tissue regeneration (26-30).

In a number of previous studies, PRF was used in combination with other bone substitute materials to realize its osteogenic effects $(19,24,25)$, but it has been uncommon to use it separately for tooth extraction site preservation. In the present study, PRF was used alone in the extraction socket as a biological material.

From clinical, radiological and histological perspectives, alveolar bone preservation and bone regeneration in the experimental group were comprehensively assessed. The results were compared with naturally healing tooth sockets. The purpose of the present study was to explore the feasibility of using PRF alone in preserving tooth extraction sites and providing a theoretical basis for its clinical practice, which may ultimately maintain an adequate quantity of bone for implant surgery.

\section{Materials and methods}

Case selection. A total of 28 patients were included in the present study. They were outpatient patients from the Department of Stomatology in East Hospital Affiliated to Tongji University (Shanghai, China) and were selected between July 2015 and March 2017. Patient characteristics from both groups are presented in Table I. Prior to surgery personal data was collected and clinical trial specialist medical records were completed. Patients gave informed written consent and were included in the experimental or control group ( $n=14$ each) according to their wishes. The experimental protocol was reviewed and approved by the Ethics Committee of East Hospital Affiliated to Tongji University.

The inclusion criteria were as follows: i) Aged from 20 to 40 years, ii) upper and lower mandibular molars diagnosed as fractured tooth or could not be retained for other reasons, as these patients required implant surgery necessarily to repair their missing teeth; iii) general good health and no uncontrolled systemic disease; and iv) compliant patients. The presents study recruited male and female patients in order to balance the sexes.

The exclusion criteria were as follows: i) Tooth extraction contraindications; ii) severe periodontal disease, as patients with serious absorption of alveolar bone and alveolar bone defects in tooth extraction sites were excluded; iii) implant surgery contraindications, such as hypertension without good control, diabetes, heart disease and other systemic diseases, and bone metabolic diseases; iv) smoking and alcoholism; and v) severe bruxism.

Preparation of radiation guide. Upper and lower mandibular research models were prepared prior to tooth extraction. The crown of the target tooth was abraded from the plaster model (Fig. 1A and B). The radiation guide plate was made from self-curing plastic. The guide covered half of both adjacent teeth was removed after the self-curing plastic had hardened. The radiation guide plate was carefully polished to make it smooth. Subsequently, a 1-mm zirconium bead was placed in the center of the guide and fixed (Fig. 1C). The guide was then placed in the model to ensure that it was appropriate (Fig. 1D and E).

Preparation and structure of PRF membrane. Following the provision of written informed consent, two samples of venous blood ( $9 \mathrm{ml}$ in each) were collected from patients in the experimental group 5 min prior to tooth extraction. The test tubes used did not contain any anticoagulant. The blood samples were extracted in strict accordance with the PRF manufacturing process (31). The venous blood was rapidly centrifuged at $400 \mathrm{x} \mathrm{g}$ at room temperature for $10 \mathrm{~min}$ using a specific table centrifuge, Hettich ${ }^{\circledR}$ Universal 320 (Andreas Hettich GmbH \& Co.KG, Tuttlingen, Germany) and kept aside for 3-5 min. The sample was then divided into three layers: The bottom layer of red blood cell (RBC) debris, the middle layer of PRF gel and the top layer of supernatant. The middle layer was removed using sterile tweezers, and the RBC layer was removed from the PRF gel. Finally, the PRF gel was placed in the PRF box (Process for PRF, Nice, France) for $10 \mathrm{sec}$ under light pressure. As a result, the tough and elastic PRF membrane was obtained. The PRF membrane was immediately fixed with $4 \%$ paraformaldehyde for $24 \mathrm{~h}$ at room temperature, dehydrated in increasing gradients of alcohol, subjected to critical point drying and gold sputter-coated to observe the ultrastructure. Samples were then viewed and images were captured using scanning electron microscopy (Quanta 250; FEI; Thermo Fisher Scientific, Inc., Waltham, MA, USA). 
Table I. Patient demographics.

\begin{tabular}{lccccccc}
\hline & & \multicolumn{2}{c}{$\begin{array}{c}\text { Extracted tooth position, } \\
\text { position, } n\end{array}$} & & \multicolumn{2}{c}{$\begin{array}{c}\text { Classification of extracted } \\
\text { tooth, } n\end{array}$} \\
Group & $\begin{array}{c}\text { Sex ratio, } \\
\text { female:male }\end{array}$ & $\begin{array}{c}\text { Age, years } \\
\text { (mean } \pm \text { SD) }\end{array}$ & $\begin{array}{c}\text { Maxillary } \\
\text { molar }\end{array}$ & $\begin{array}{c}\text { Mandibular } \\
\text { molar }\end{array}$ & & $\begin{array}{c}\text { Fractured } \\
\text { tooth }\end{array}$ & $\begin{array}{c}\text { Residual root } \\
\text { and crown }\end{array}$ \\
\hline PRF & $6: 8$ & $33.2 \pm 3$ & 7 & 7 & & 9 & 5 \\
Control & $8: 6$ & $34.6 \pm 4$ & 6 & 8 & & 8 & 6 \\
\hline
\end{tabular}

SD, standard deviation; PRF, platelet-rich fibrin.
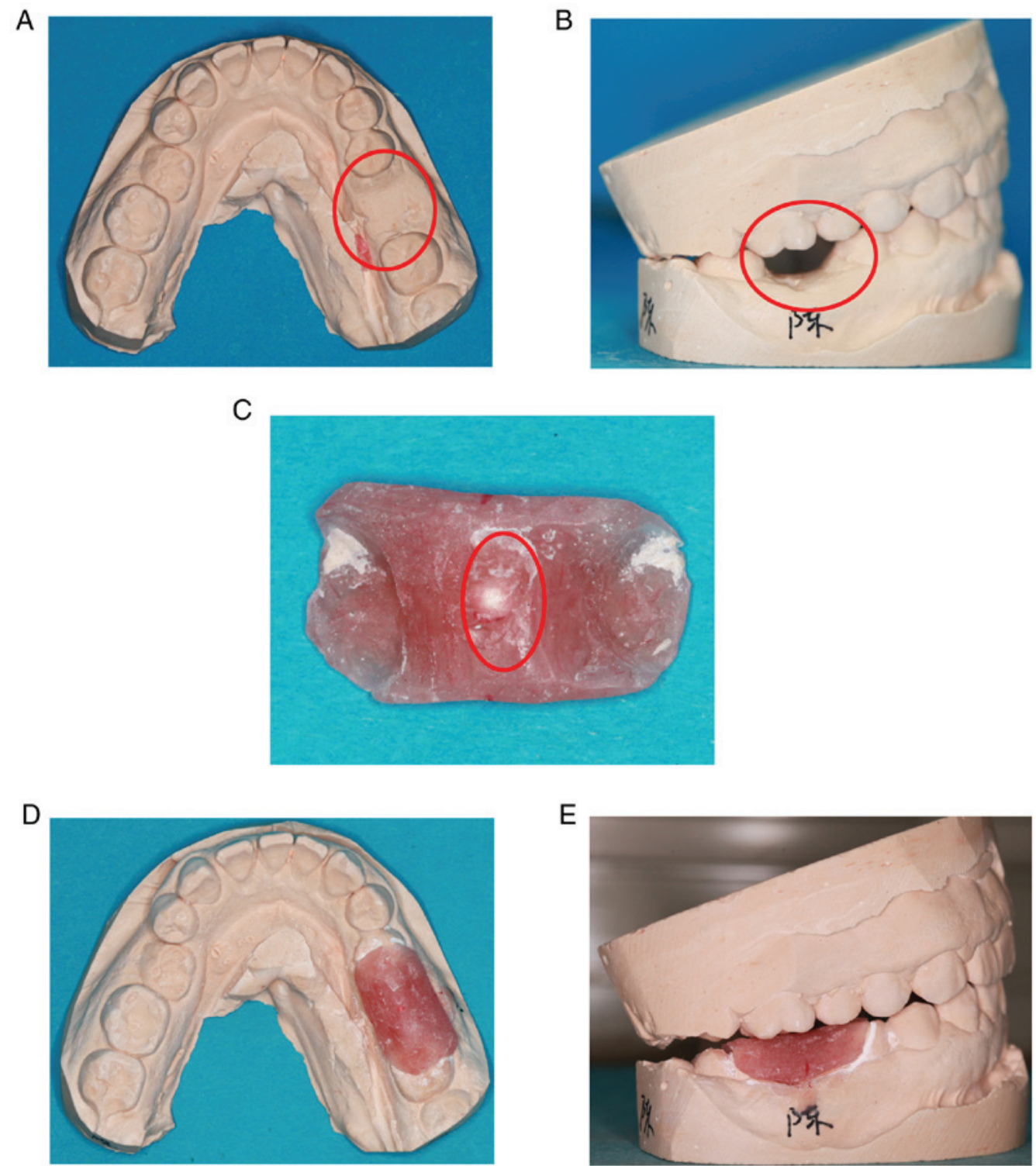

Figure 1. Preparation of radiation guide. (A and B) The diseased tooth was abraded from the plaster model. (C) Radiation guide produced using self-curing plastic with zirconium beads in the middle. (D and E) The radiation guide placed into the plaster model.

Minimally invasive tooth extraction. Patients were asked to undergo an oral panoramic radiograph prior to tooth extraction; this procedure was performed using a using an Orthophos XG 5 (Dentsply Sirona, York, PA, USA). An oral examination was performed, and local and systemic conditions were assessed. All patients were treated with the non-flap minimally invasive extraction technology following the use of $1.8 \mathrm{ml}$ of $4 \%$ articaine hydrochloride with epinephrine $(1: 100,000)$ by subperiosteal infiltration (Ubistesin ${ }^{\mathrm{TM}} 1 / 100,000 ; 3 \mathrm{M}$ ESPE AG, Seefeld, Germany) as local anesthesia. All inflammatory 
A

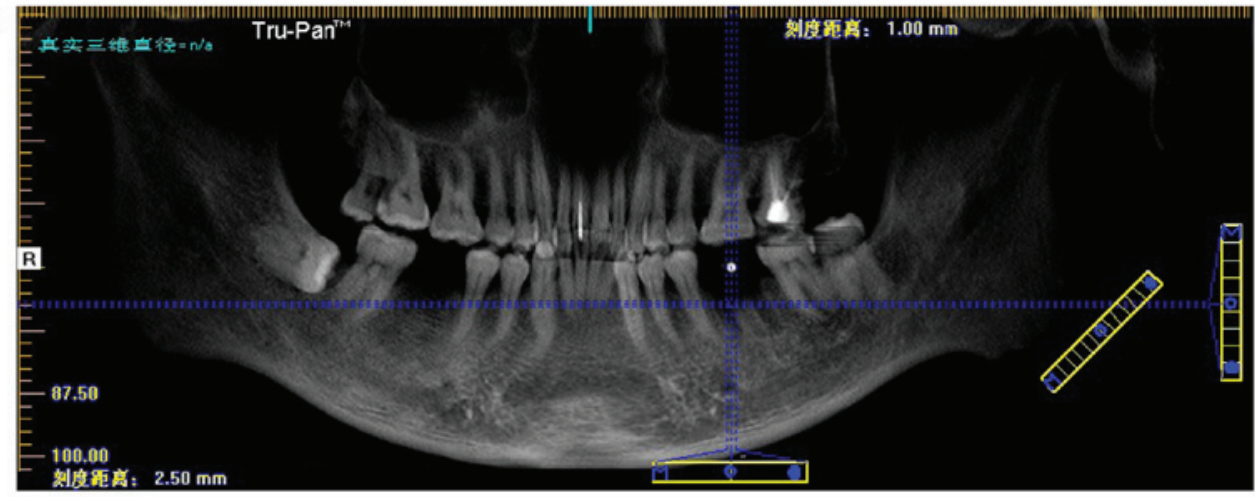

B

C
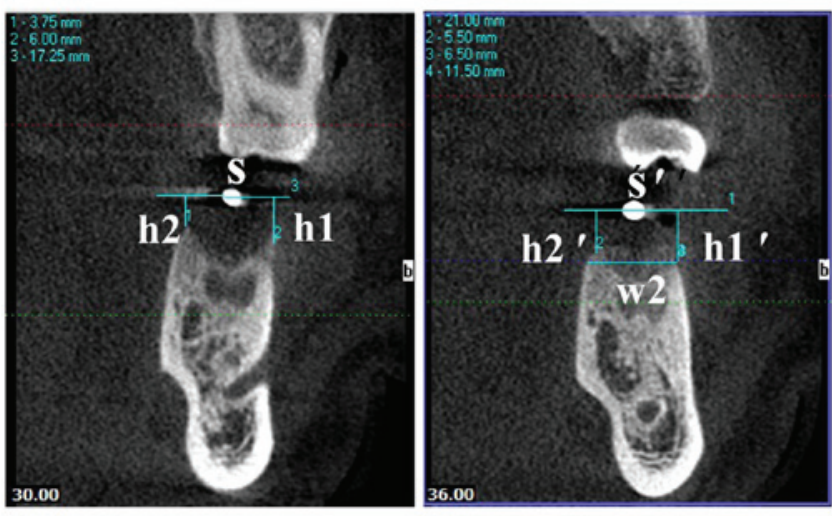

D

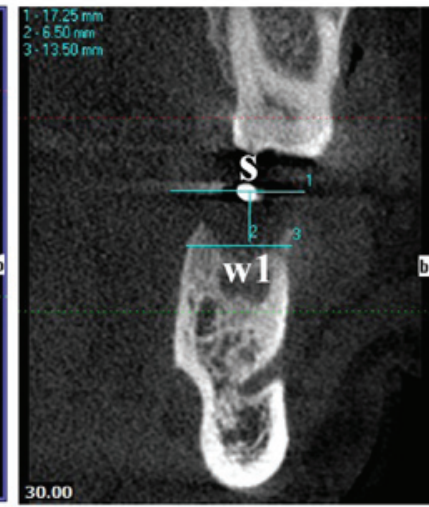

Figure 2. CBCT data measurement. (A) The horizontal blue line coincided with the maxillary or mandibular alveolar crest, and the vertical blue line passed through the center of the position of the zirconium beads. (B) The CBCT image obtained at T1, where S, h1 and h2 were obtained. (C) On the CBCT at 3 months following tooth extraction, the horizontal line $\mathrm{S}^{\prime}$ and the vertical distance $\mathrm{h} 1^{\prime}$ and $\mathrm{h} 2$ ' were obtained by the same method. w2 was measured by drawing a horizontal line through the buccal or lingual alveolar ridge vertex position. (D) w1 on the CBCT image of T1 was obtained at the same distance from the vertebral alveolar ridge. CBCT, cone-beam computed tomography; T1, immediately following tooth extraction; S, line through center of zirconium bead; $\mathrm{h} 1$, vertical distance of the buccal alveolar crest to the line; h2, vertical distance of the palate/lingual alveolar crest to the line; w1, buccal-lingual alveolar ridge width at $\mathrm{T} 1$; w2, buccal-lingual alveolar ridge width at $\mathrm{T} 2$.

granulation tissue was then removed from the extraction socket. In the experimental group, PRF membranes were cut into a suitable size and implanted into the extraction socket following tooth extraction. The gum surrounding the tooth socket was loosened and sutured following extraction. If the gum was not completely sutured, one entire PRF membrane would be placed at the base of the extraction socket and another PRF membrane covered the first membrane. Therefore the first PRF membrane would be isolated from the rest of the mouth. The extraction sockets in the control group were left for natural healing after conventional suturing. The gauze was held for 30-40 min following tooth extraction in the experimental and control groups. Subsequently, three-dimensional reconstruction of cone-beam computed tomography (CBCT) was taken with the radiation guide that was previously prepared. CBCT was taken with the respective radiating plate again at 3 months following tooth extraction. Postoperative response following extraction and local soft tissue healing condition were assessed at 3 and 7 days following tooth extraction. The healing of local gingival tissue was recorded at 1 and 3 month following tooth extraction.

CBCT scan. Scans were performed at 30-40 min (T1) and 3 months (T2) following tooth extraction. Patients' posterior teeth contacted with the pre-prepared radiating guide, and the upright posture was taken. The operator was located in front of the patient and adjusted the CBCT and the patient's positions so that the horizontal alignment light overlapped the lips and vertical calibration of the light in front of the condyle for 1.5 inches. All procedures were performed by the same physician with the same CBCT machine, and the following scan uniform parameters were set: X-ray exposure settings, Voltage $=120 \mathrm{~V}$; Current $=5 \mathrm{~mA}$; scan window, diameter $=16 \mathrm{~cm}$, height $=13 \mathrm{~cm}$, 25 stereo pixels, $26.9 \mathrm{sec}$; image matrix size $=640 \times 640$ pixels; and layer sweep thickness $=0.250 \mathrm{~mm}$. All data were measured by the same radiologist in department of Stomatology and analyzed by a blinded surveyor. Each measurement was taken in triplicate to reduce the measurement error. Finally, the mean value was adopted in the final statistical analysis. Data measurement was completed using eXamVision software (version 1.9; KaVo Dental GmbH, Biberach, Germany). Analysis was performed as described in the following paragraph.

The blue line on the panorama view of the implant screen was dragged so that the horizontal blue line coincided with the maxillary or mandibular alveolar crest, and the vertical blue line passed through the center of the positioning of the zirconium beads. The sweep thickness and gap were adjusted to $1 \mathrm{~mm}$ (Fig. 2A). The blue line was positioned at the gap of the tooth on the CBCT image immediately obtained following tooth extraction (T1). A horizontal line $\mathrm{S}$ was drawn through the center of the zirconium beads. The vertical distance of the buccal alveolar crest to the line was recorded as h1, and the 
A

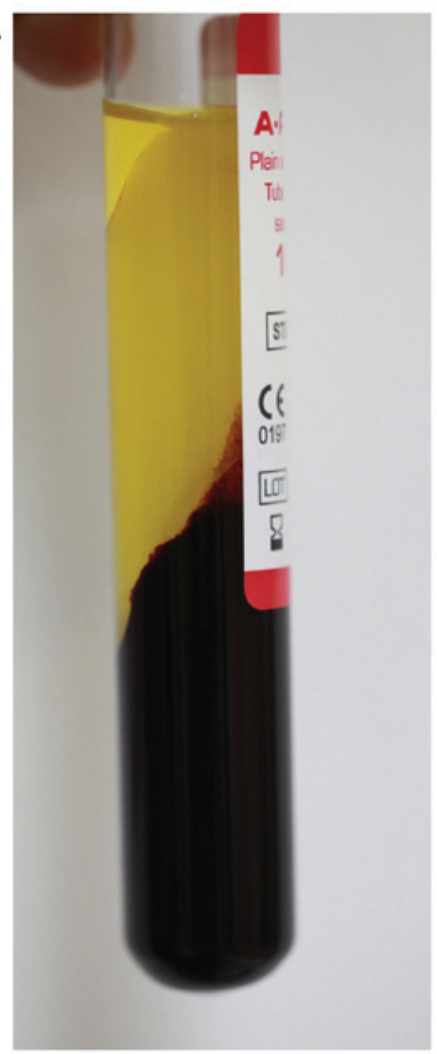

B

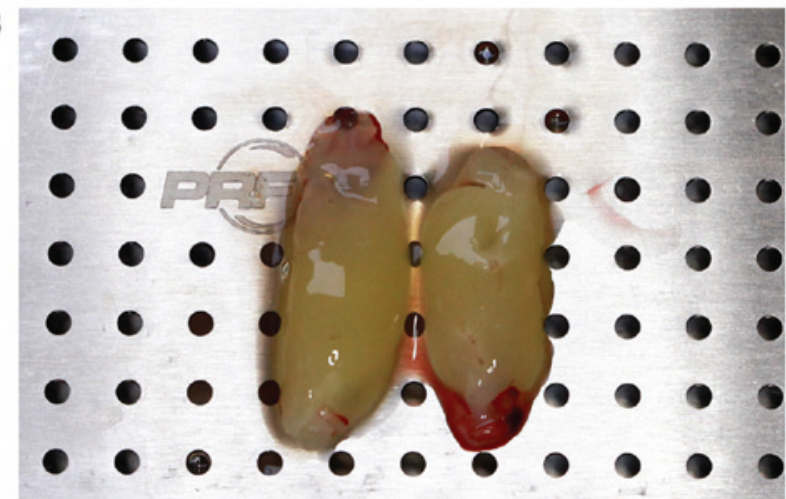

C

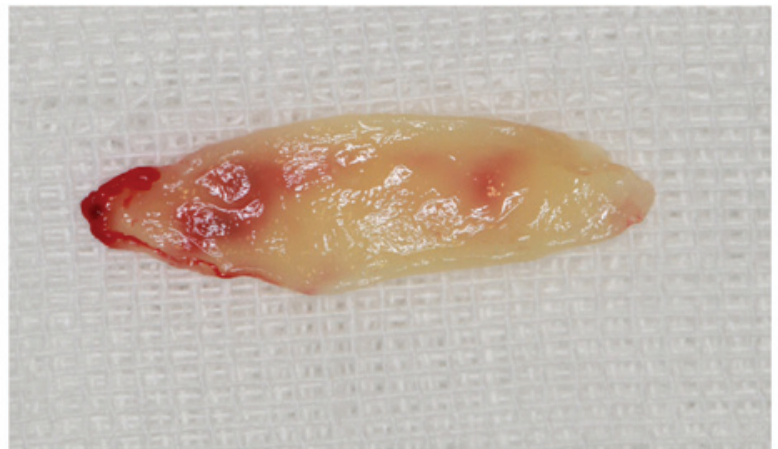

Figure 3. Appearance of PRF. (A) Three fractions of blood generated by centrifugation: PPP, PRF and red blood cells. (B) PRF gel with translucent jelly structure and smooth surface, was durable and elastic. (C) PRF membrane. PRF, platelet-rich fibrin; PPP, platelet-poor plasma.

palate/lingual alveolar crest to the line as h2. The horizontal line $\mathrm{S}^{\prime}$ and the vertical distance $\mathrm{h} 1^{\prime}$ and $\mathrm{h} 2$ ' were obtained by the same method on the CBCT of T2. The buccal-lingual alveolar ridge width w2 was measured by drawing a horizontal line through the buccal or lingual alveolar ridge vertex position (a higher position was chosen). The buccal-lingual alveolar ridge width w1 on the $\mathrm{CBCT}$ image of $\mathrm{T} 1$ was obtained at the same distance from the vertebral alveolar ridge (Fig. 2B-D). The following differences were defined: the variation value of the height of buccal alveolar crest=h1'-h1; the variation value of the height of lingual/palatal alveolar crest=h2'-h2; the variation value of the width of alveolar crest=w1-w2. All differences were given as absolute values.

Implant surgery. Blood routine check was performed prior to surgery. Implant surgery was performed under local anesthesia with Ubistesin 1/100,000, following provision of preoperative informed consent. Initially, the flap was cut in the center of the gums, and granulation tissue was removed. A height of 3-4 $\mathrm{mm}$ alveolar bone was then obtained in the center of the planting site using a ring drill to prepare hard tissue sections, the inner diameter and the outer diameter of which were 3.2 and $4.0 \mathrm{~mm}$, respectively. Bone samples (diameter, $3 \mathrm{~mm}$; height, 3-4 $\mathrm{mm}$ ) were separated and fixed in Million's solution (Sinopharm Group Co., Ltd., Shanghai, China) for 3 days (daily exchange) at room temperature, rinsed in flowing tap water overnight, dehydrated using ethanol (40, 70,95 and $100 \%$ ) every two day, soaked in xylene for 1 day at room temperature, and embedded in methyl methacrylate. The samples were cut into $10-\mu$ m-thick sections following

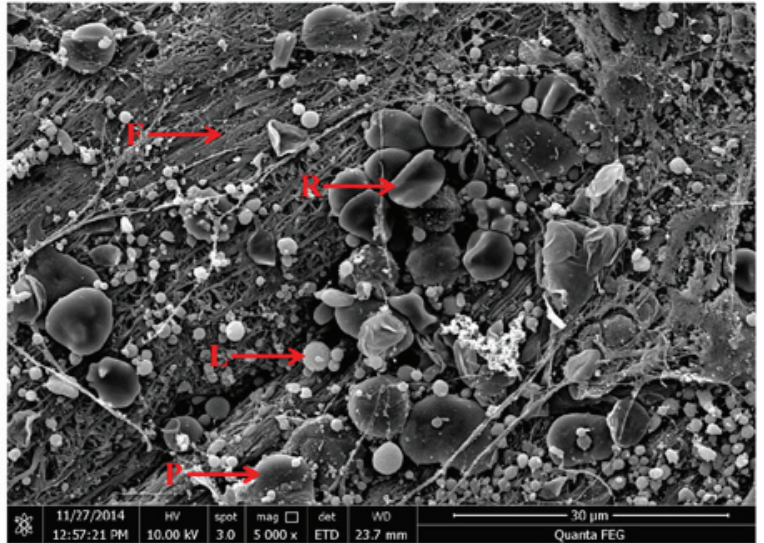

Figure 4. Scanning electron microscopy image of platelet-rich fibrin (magnification, x5,000). Many fibrin fibers assembled to form a tight three-dimensional fibrin network structure. Numerous platelets, leukocytes, and red blood cells were embedded in the network. F, fibrin fibers; P, platelets; L, leukocytes; $\mathrm{R}$, red blood cells.

solidifying in a vacuum. The sections were stained with Goldner's trichrome at room temperature. Goldner's Trichrome staining procedure was as follows: The sections were stained with Hematoxylin-Ferric Chloride Dye for $7 \mathrm{~min}$, rinsed with tap water two times, stained with Ponceau acid fuchsin for 7 min, rinsed with $1 \%$ acetic acid for 1 min, incubated with $1 \%$ phosphomolybdic acid for $6 \mathrm{~min}$, rinsed with $1 \%$ acetic acid for $1 \mathrm{~min}$, incubated with $0.1 \%$ bright green solution staining for several minutes, rinsed with $1 \%$ acetic acid for $1 \mathrm{~min}$. Finally, the stained sections were dried at $37^{\circ} \mathrm{C}$ overnight 


\section{A}
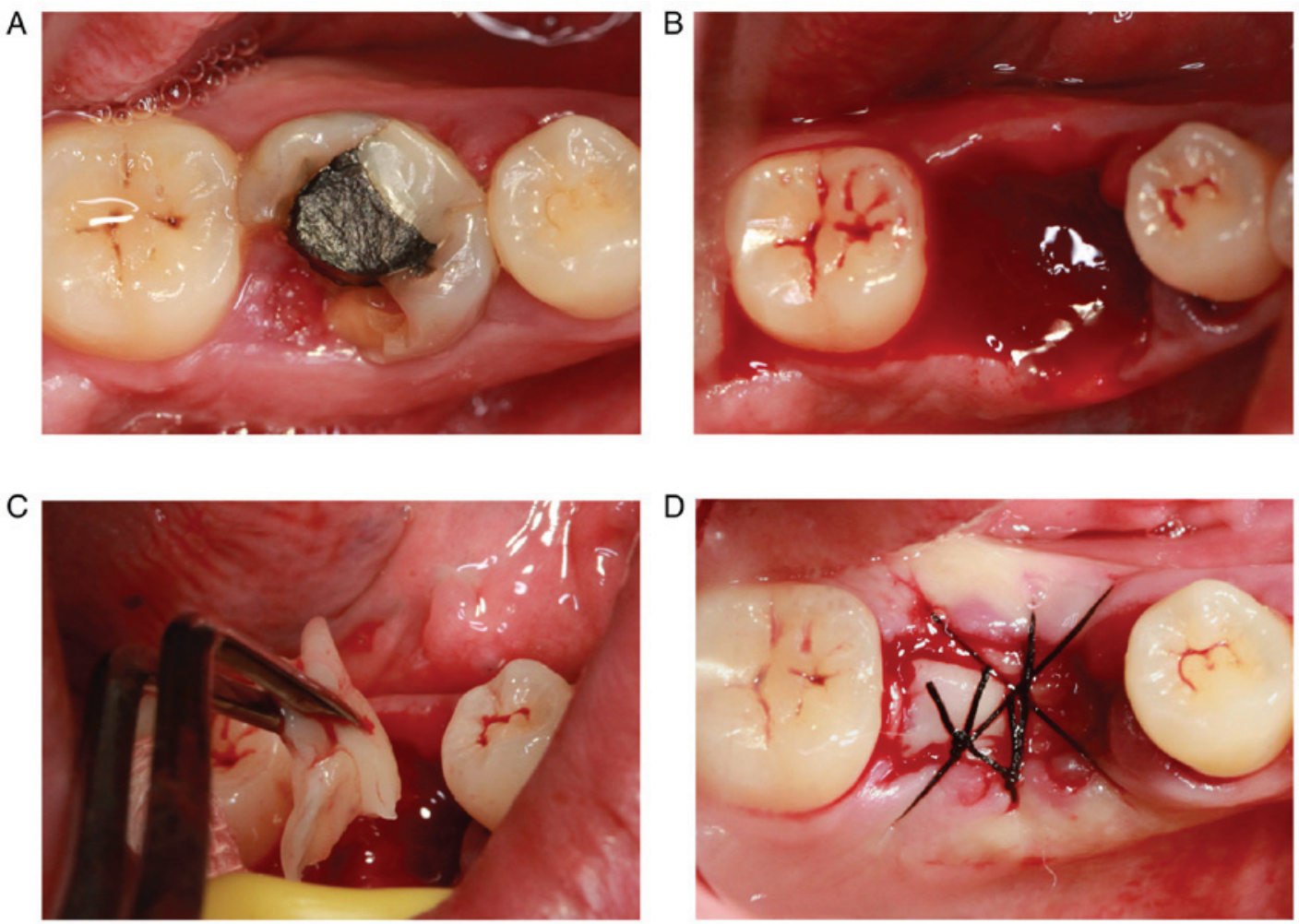

Figure 5. Process of minimally invasive tooth extraction and the application of PRF. (A) Clinical manifestations of the tooth prior to extraction. (B) Extraction socket. (C) Application of PRF in the extraction socket. (D) Suturing of the extraction socket. PRF, platelet-rich fibrin.

and sealed with neutral gum. Then all stained sections were observed under a light microscope and photographed using Leica Application Suite software (version 4.5) (both Leica Microsystems GmbH, Wetzlar, Germany). The novel bone formation of the two groups was analyzed using SimplePCI 5.2.1 (Compix, Inc., Cranberry, PA, USA), and the osteoid area/tissue area (OAr/TAr) was calculated. The corresponding types of implants (NobelReplace Tapered Groovy; Nobel Biocare Services AG, Zürich, Switzerland) were implanted following the step-by-step expansion of the hole. The healing abutment was screwed into the implant with a torque of 15 Ncm. Finally, the wound was sutured using 4-0 absorbable sutures.

Statistical analysis. Data analysis was performed using SPSS version 20.0 software (IBM Corp., Armonk, NY, USA). Data are presented as the mean \pm standard deviation. Statistical differences between the groups were determined using one-way analysis of variance. $\mathrm{P}<0.05$ was considered to indicate a statistically significant difference.

\section{Results}

PRF membrane preparation and its structure. Venous blood exhibited three fractions following centrifugation: The lower layer of RBCs, the middle layer of PRF and the upper layer of platelet-poor plasma (Fig. 3A). The surface of the PRF layer was smooth and flexible, and contained a light yellow liquid (Fig. 3B). PRF gels were extruded from the liquid component by means of a lamination tool to form a membranous structure with durability and flexibility (Fig. 3C). As demonstrated in the scanning electron micrograph (Fig. 4), fibrin fibers tended to form a tight three-dimensional fibrin network structure. RBCs were trapped within this fibrin matrix. Leukocytes appeared as spherical structures with an irregular surface. The platelets were often enmeshed in the fibrin network but sometimes appeared as aggregates.

Minimally invasive extraction and the process of PRF application. A representative case was chosen to demonstrate this process. The first molar of the patient (a 28-year-old female) was largely absent and the defect of the crown was $<3 \mathrm{~mm}$ of the subgingival tissue (Fig. 5A). Therefore, the tooth could not be retained following a comprehensive assessment. The broken teeth were removed via minimally invasive tooth extraction surgery (Fig. 5B). The PRF membranes prepared were cut into a suitable size and implanted into the extraction socket following removal of the granulation tissue in the alveolar fossa (Fig. 5C). Finally, the tooth extraction socket was sutured (Fig. 5D). The extraction sockets in the control group were left for natural healing.

Postoperative follow-up results. The patients in the control group reported feeling pain within 3 days following tooth extraction. The pain had disappeared after 1 week. Patients in the experimental group reported mild pain the day after surgery. The pain was not obvious on the second day. The majority of patients felt no pain or discomfort after 3 days. The PRF membrane was clearly visible at 7 days following tooth extraction, and PRF was not fully absorbed. The tooth socket was partially closed, and the local gingival tissue had no symptoms of swelling and infection (Fig. 6A). The local 

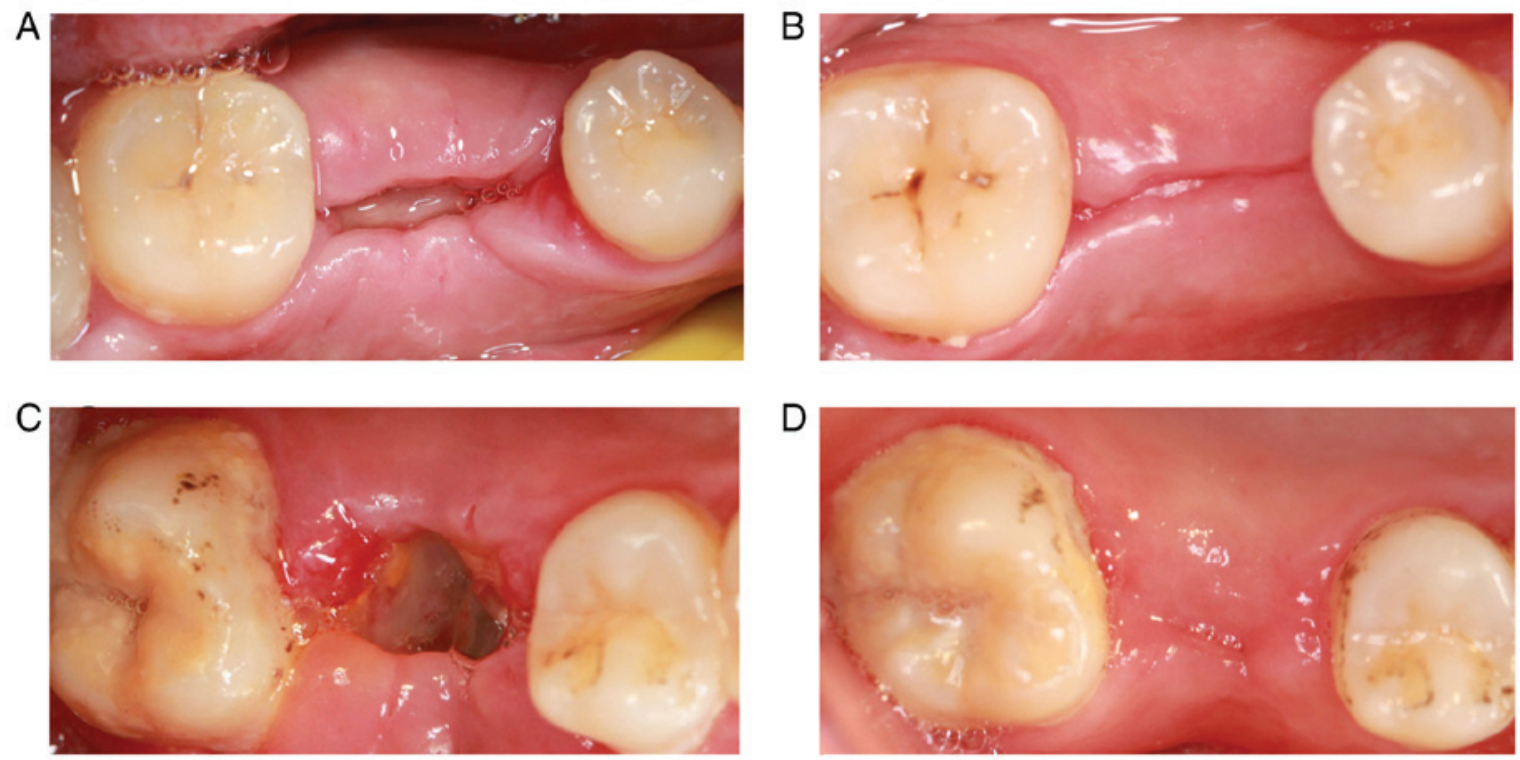

Figure 6. Healing of local gingival tissue following tooth extraction. Gingival tissue of the experimental group at (A) 7 days and (B) 3 months following tooth extraction. Gingival tissue of the control group at (C) 7 days and (D) 3 months following tooth extraction.

gingival tissue had healed well by 3 months following tooth extraction, but a gap was still visible (Fig. 6B). The majority of tooth extractions were not closed at 7 days following tooth extraction in the control group. Also, the white pseudomembrane was observed in the tooth extraction, and the local gingival tissue was slightly swollen (Fig. 6C). The tooth extractions were closed by 3 months following tooth extraction. No signs of infection were observed in the local gingival tissue (Fig. 6D).

CBCT analysis results. The variation value of the height of buccal alveolar crest and lingual/palatal alveolar crest, and the variation value of the width of the alveolar crest in the two groups were measured as detailed above. The variation value of the buccal alveolar crest was markedly lower in the PRF group $(1.6000 \pm 1.46416)$ compared with the control group (2.8000 \pm 1.81487 ; Fig. 7). The variation value of the height of the lingual/palatal alveolar crest was also lower in the PRF group $(1.0000 \pm 0.70711)$ compared with the control group (2.0500 \pm 1.29180 ; Fig. 7). The variation value of the width of the alveolar crest was also lower in the PRF group (1.0500 \pm 0.77862$)$ compared with the control group

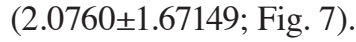

Implantation surgery. The implantation surgery was performed 3 months following tooth extraction. After cutting the flap, the alveolar bone surface was found to be smooth, with no obvious bone depression, and granulation tissue was rare (Fig. 8A). During the operation, the alveolar bone with a diameter of $2 \mathrm{~mm}$ and length of $\sim 3 \mathrm{~mm}$ was drilled with a circular drill at the planting center (Fig. 8B-E). The healing abutment was inserted following implantation of the implant. Finally, the wound was sutured (Fig. 8F).

Histological analysis of new bone formation in the two groups. The alveolar bone obtained during the implant surgery was prepared for the hard tissue sections. Following

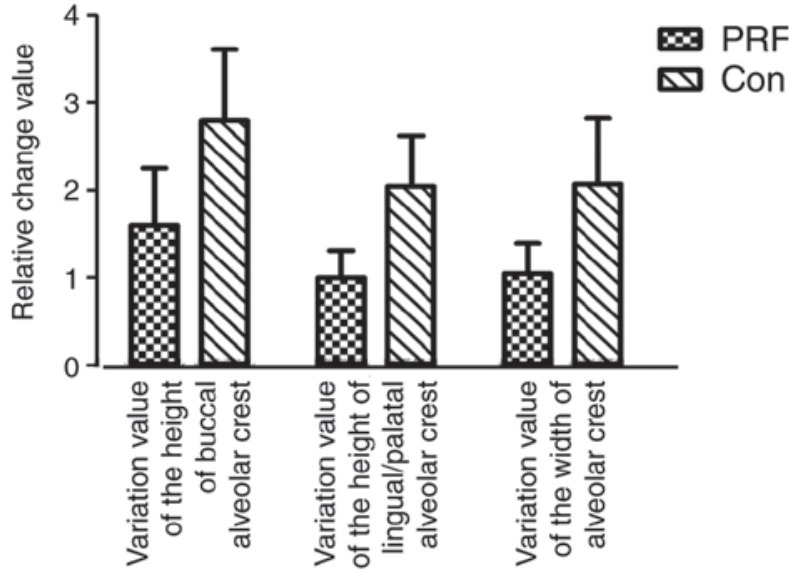

Figure 7. Variation values of the buccal and lingual/palatal alveolar crest height and the alveolar crest width in the two groups. Data are presented as the mean \pm standard deviation $(n=14)$. PRF, platelet-rich fibrin; Con, control.

Goldner's trichrome staining, the new bone formation in the PRF group (Fig. 9A and B) was relatively abundant compared with that in the control group (Fig. 9C and D) when observed under the microscope. OAr/TAr in the experimental group $(9.7624 \pm 4.0121 \%)$ was significantly greater than that in the control group $(2.8056 \pm 1.2094 \%$; $\mathrm{P}<0.01$; Fig. 10).

\section{Discussion}

PRF was used alone for alveolar fossa in the present study. The aim was to retain as much alveolar bone as possible; therefore, minimally invasive surgery was adopted to remove the teeth to minimize the alveolar bone destruction caused by tooth extraction. A previous study confirmed that if flap surgery is used in extraction surgery, the opened periosteal flap blocks the blood supply of the buccal bone wall, further increasing buccal bone wall absorption (32). In addition, the 

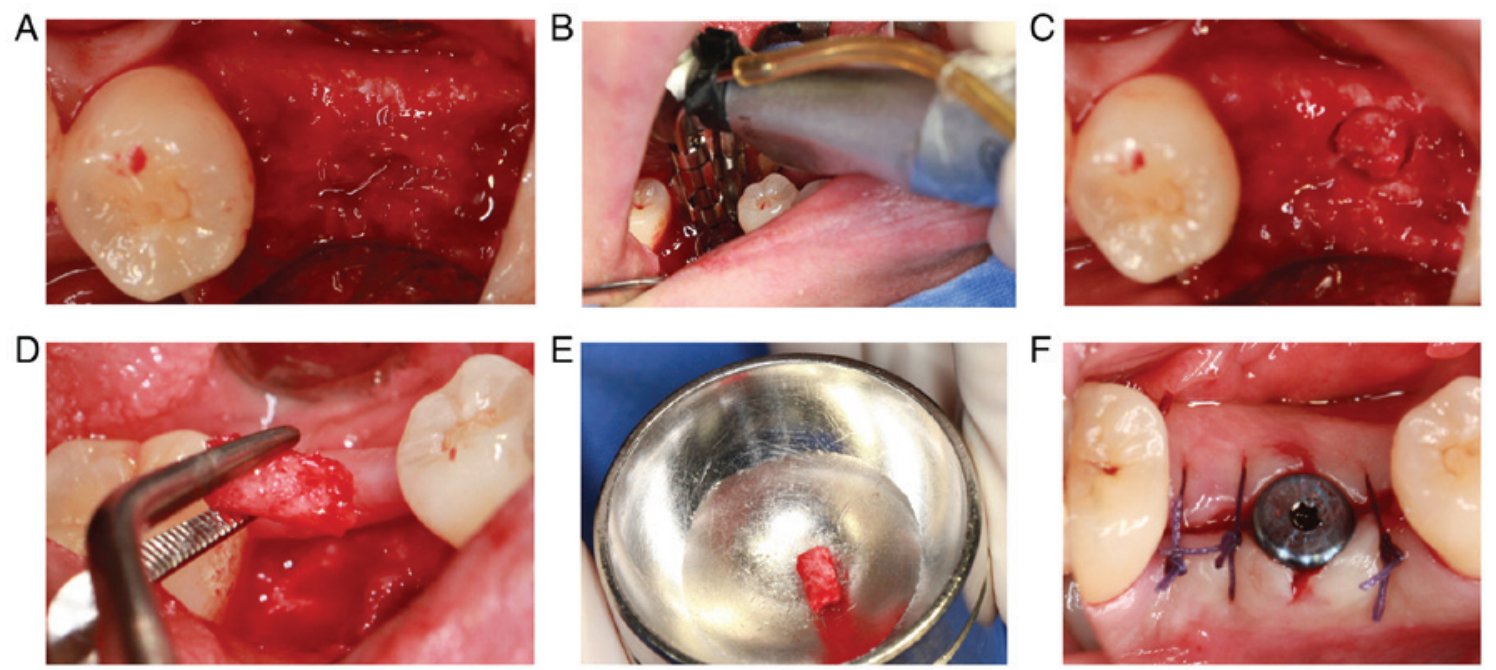

Figure 8. Implant surgery of the experimental group. (A) The surface of the alveolar crest following the mucoperiosteal flap was completed. (B-E) Alveolar bone was collected via annular drilling. (F) The implant surgery was completed, and the soft tissue was sutured.
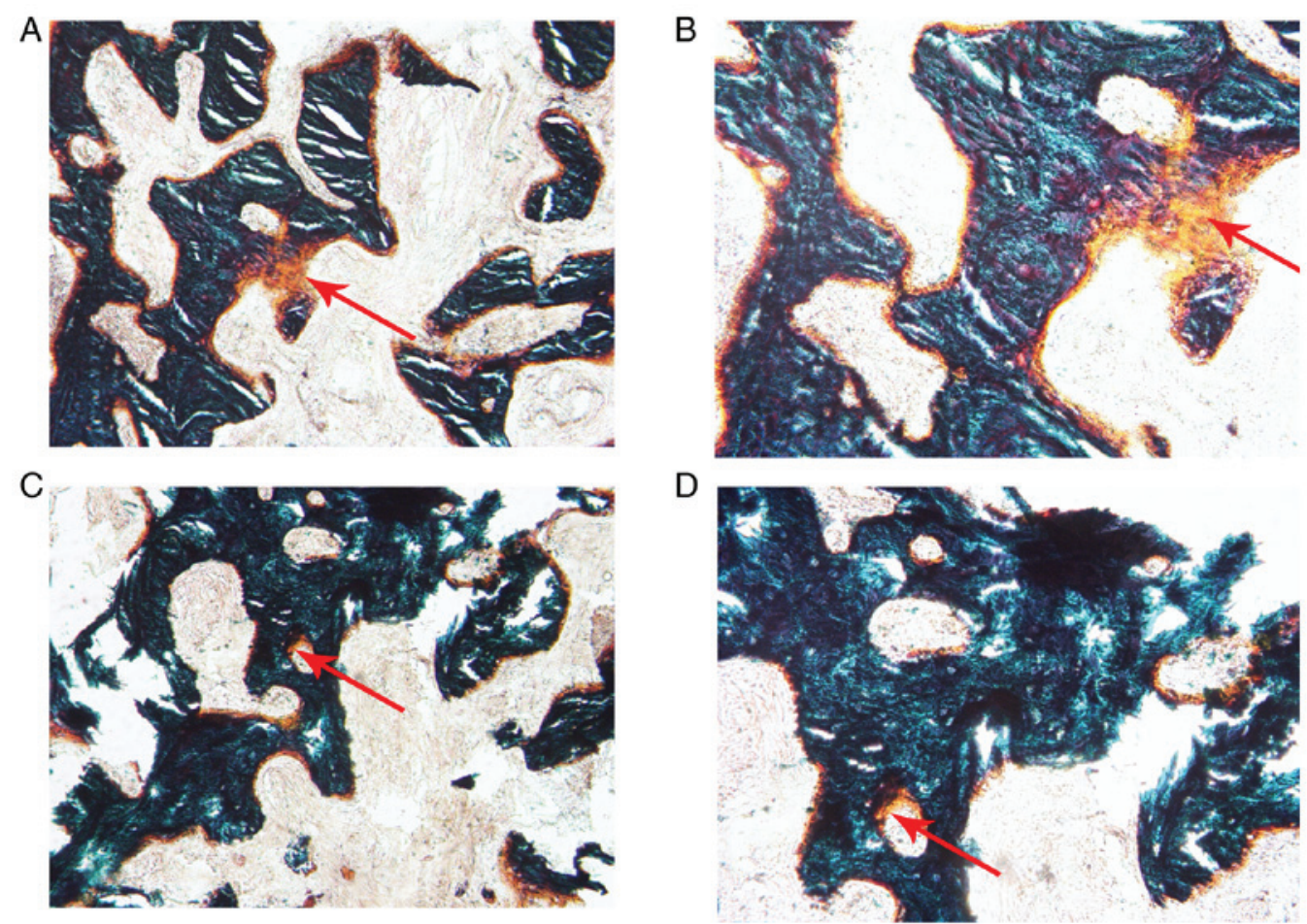

Figure 9. Observation of the novel bone following Goldner's trichrome staining. The formation of novel bone was more abundant in the experimental group than in the control group. (A) Experimental group (magnification, x100); (B) experimental group (magnification, x200); (C) control group (magnification, $\mathrm{x} 100$ ); and (D) control group (magnification, x200). Red arrows indicate novel bone.

use of flap surgery may increase the risk of gum gingival recession and reduce the width of keratosis (33). Therefore, flap surgery was not performed in the present study when the teeth were removed. However, CBCT scanning was performed immediately following extraction, instead of prior to tooth extraction, to reduce the damage of the alveolar fossa caused by the tooth extraction surgery. The following measures were taken to retain the PRF in a relatively confined space: The gum surrounding the tooth socket was loosened and sutured following extraction; if the gum was not completely sutured, the entire PRF membrane would be covered, to ensure that the PRF was relatively isolated from the rest of the mouth.

Patients in the experimental group exhibited a lesser postoperative reaction compared with the patients in the control group, in terms of clinical manifestations. However, these indicators may not serve as an ideal theoretical basis because of variations between individuals. Furthermore, the healing of soft tissue in the experimental group was improved compared with that in the control group, and no symptoms of inflammation were observed, indicating that PRF enhanced the healing of soft tissue, which was consistent with previous 


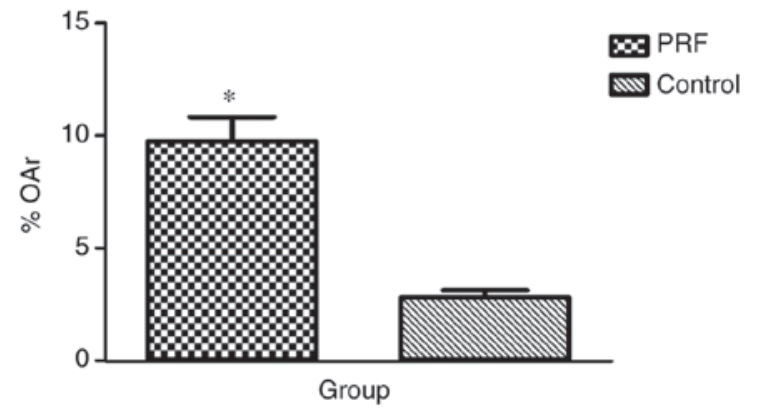

Figure 10. \% OAr, which corresponds to the percentage of novel bone formation area in the experimental and control groups. Data are presented as the mean \pm standard deviation $(\mathrm{n}=14) .{ }^{*} \mathrm{P}<0.01$ vs. control. PRF, platelet-rich fibrin; OAr, osteoid area.

studies $(20,28,30)$. The present results regarding enhanced soft tissue healing were due to the properties of PRF, as detailed above.

In the present study, histological analysis demonstrated that the novel bone formation in the PRF group was significantly increased compared with that in the control group; which suggested that PRF is able to stimulate bone regeneration. It has previously been reported that bone graft healing is a sequential process that consists of inflammation revascularization, osteogenesis remodeling and incorporation into the host skeleton to form a mechanically efficient structure, wherein the ingrowth, proliferation and differentiation of osteoblasts occurs during the initial 14 days $(34,35)$. Furthermore, it has also been demonstrated that PRF released autologous growth factors gradually and expressed a durable effect on the proliferation and differentiation of rat osteoblasts in vitro. The maximal promoting effect of PRF occurred at day 14 (21). These findings suggest that the growth factors contained in PRF may serve a role in promoting bone regeneration, over a prolonged period of time.

In the present study, the changes in alveolar bone height and bone mineral density were analyzed using CBCT quantitative analysis software, whose radiation dose was relatively low and the repeatability was strong. All procedures were performed by the same doctor, and the patient position was maintained when taking CBCT scans at different time points, to reduce human error. Furthermore, the measurement data had a clear reference mark through the preparation of the radiation guide, thereby reducing the error in data measurement. Changes in alveolar bone have previously been studied through the root tip; however, the results were affected by the angle of shooting, position of the patient, location of the film and magnification of the image (29). The results of imaging analysis indicated that the variation values of the height of the buccal alveolar crest and lingual/palatal alveolar crest, and the variation value of the width of the alveolar crest were markedly lower in the experimental group than in the control group. However, the differences between the two groups were not statistically significant. Therefore, it could not be concluded that PRF was effective in reducing alveolar bone resorption and promoting bone formation in the extraction socket. This finding was in accordance with a previous clinical study (36). It is presumed that the effect of bone regeneration was limited and could not maintain an alveolar ridge shape following tooth extraction because of the low levels of the cytokines in PRF. However, the present study corresponded with previous studies where the loss of buccal marginal bone level was more pronounced on the buccal than on the lingual counterpart (37-39). Conversely, the present findings were different from the results of a number of other previous studies; in the present study the alveolar bone was preserved, which was not the case in previous studies (26-30). Possible reasons are as follows: i) The application of the radiation guide promoted the accuracy of the measurement; ii) examining the dimensional changes of the alveolar ridge via CBCT ensured the reproducibility of the experiment and reduced human error; iii) histological analysis of new bone formation was performed; and iv) the teeth used in the present study were not single-rooted, as are used in many other studies. Socket anatomy is an important disturbing factor because of a variety in width and height of the socket, thickness and densities of osseous plates, presence of fenestrations and dehiscence, periodontal biotype, form (single or multi rooted) and location of the socket (maxilla or mandible). Therefore, it is difficult to quantify the real role of a graft in the limitation of osseous resorption.

In the present study, patients were recruited by following the inclusion and exclusion criteria. Therefore, the number of patients was relatively small. The majority of teeth included in the present study were broken, or had residual roots or crowns (which could not be retained by assessment), resulting in the reduction of sample size. Furthermore, experimental protocols were interfered by a number of factors. Inevitably patients were lost in the follow-up, resulting in the loss of corresponding data and insufficiency of effective data. If some of the follow-up data of a patient was missing, the patient was removed from the final analysis. In the present study, 4 patients (2 patients in each group) were lost in the follow-up; their information was not included in the statistical analysis. Therefore, it was difficult to demonstrate the final conclusion. However, the osteogenesis effect of Bio-Oss is internationally recognized. Previous studies have demonstrated that combining Bio-Oss and PRF in clinical applications preserves alveolar bone and also shortens healing time $(19,40)$. However, no conclusive evidence is currently available regarding the proportion required of both, which may be the focus of future research.

In conclusion, PRF membrane used in the extraction sockets was demonstrated to promote local soft tissue healing of gums and reduce postoperative pain response; however, the effect of PRF to reduce alveolar bone resorption was not significant. However, PRF was able to increase the quality of the novel bone and enhance the rate of bone formation due to the concentration of growth factors. Despite certain limitations, the present study demonstrated the potential for preserving the alveolar ridge following tooth extraction. A larger sample size may be required to obtain a conclusive result of the bone regeneration in extraction sockets using the PRF membrane.

\section{Acknowledgements}

The present study was supported by the Science and Technology Development Exhibition Innovation Fund of Pudong New District (Shanghai, China; grant no. PKJ2013-Y15). 


\section{References}

1. Wood DL, Hoag PM, Donnenfeld OW and Rosenfeld LD: Alveolar crest reduction following full and partial thickness flaps. J Periodontol 43: 141-144, 1972.

2. Ten Heggeler JM, Slot DE and Van der Weijden GA: Effect of socket preservation therapies following tooth extraction in non-molar regions in humans: A systematic review. Clin Oral Implan Res 22: 779-788, 2011.

3. Araujo MG and Lindhe J: Ridge alterations following tooth extraction with and without flap elevation: An experimental study in the dog. Clin Oral Implan Res 20: 545-549, 2009.

4. Wang RE and Lang NP: Ridge preservation after tooth extraction. Clin Oral Implants Res 23 (Suppl 6): S147-S156, 2012.

5. Darby I, Chen ST and Buser D: Ridge preservation techniques for implant therapy. Int J Oral Maxillofac Implants 24 (Suppl): S260-S271, 2009.

6. Bartee BK: Extraction site reconstruction for alveolar ridge preservation. Part 1: Rationale and materials selection. J Oral Implantol 27: 187-193, 2001.

7. Serino G, Biancu S, Iezzi G and Piattelli A: Ridge preservation following tooth extraction using a polylactide and polyglycolide sponge as space filler: A clinical and histological study in humans. Clin Oral Implants Res 14: 651-658, 2003.

8. Brugnami F, Then PR, Moroi H, Kabani S and Leone CW: GBR in human extraction sockets and ridge defects prior to implant placement: Clinical results and histologic evidence of osteoblastic and osteoclastic activities in DFDBA. Int J Periodontics Restorative Dent 19: 259-267, 1999.

9. Serino G, Biancu S, Iezzi G and Piattelli A: Ridge preservation following tooth extraction using a polylactide and polyglycolide sponge as space filler: A clinical and histological study in humans. Clin Oral Implan Res 14: 651-658, 2003.

10. Yilmaz S, Efeoğlu E and Kiliç AR: Alveolar ridge reconstruction and/or preservation using root form bioglass cones. J Clin Periodontol 25: 832-839, 1998 .

11. Gauthier O, Boix D, Grimandi G, Aguado E, Bouler JM, Weiss P and Daculsi G: A new injectable calcium phosphate biomaterial for immediate bone filling of extraction sockets: A preliminary study in dogs. J Periodontol 70: 375-383, 1999.

12. Wang L, Zou D, Zhang S, Zhao J, Pan K and Huang Y: Repair of bone defects around dental implants with bone morphogenetic protein/fibroblast growth factor-loaded porous calcium phosphate cement: A pilot study in a canine model. Clin Oral Implants Res 22: 173-181, 2011.

13. Howell TH, Fiorellini J, Jones A, Alder M, Nummikoski P, Lazaro M, Lilly L and Cochran D: A feasibility study evaluating rhBMP-2 absorbable collagen sponge device for local alveolar ridge preservation or augmentation. Int J Periodontics Restorative Dent 17: 124-139, 1997.

14. Wang L, Huang Y, Pan K, Jiang X and Liu C: Osteogenic responses to different concentrations/ratios of BMP-2 and bFGF in bone formation. Ann Biomed Eng 38: 77-87, 2010.

15. Dohan Ehrenfest DM: How to optimize the preparation of leukocyte- and platelet-rich fibrin (L-PRF, Choukroun's technique) clots and membranes: Introducing the PRF Box. Ora Surg Oral Med Oral Pathol Oral Radiol Endod 110: 275-280, 2010.

16. Dohan Ehrenfest DM, Del Corso M, Diss A, Mouhyi J and Charrier JB: Three-dimensional architecture and cell composition of a Choukroun's platelet-rich fibrin clot and membrane. J Periodontol 81: 546-555, 2010.

17. Li Q, Pan S, Dangaria SJ, Gopinathan G, Kolokythas A, Chu S, Geng Y, Zhou Y and Luan X: Platelet-rich fibrin promotes periodontal regeneration and enhances alveolar bone augmentation. Biomed Res Int 2013: 638043, 2013.

18. Anitua E, Alkhraisat MH and Orive G: Perspectives and challenges in regenerative medicine using plasma rich in growth factors. J Control Release 157: 29-38, 2012.

19. Choukroun J, Diss A, Simonpieri A, Girard MO, Schoeffler C, Dohan SL, Dohan AJ, Mouhyi J and Dohan DM: Platelet-rich fibrin (PRF): A second-generation platelet concentrate. Part V: Histologic evaluations of PRF effects on bone allograft maturation in sinus lift. Oral Surg Oral Med Oral Pathol Oral Radiol Endod 101: 299-303, 2006.

20. Anilkumar K, Geetha A, Umasudhakar, Ramakrishnan T, Vijayalakshmi R and Pameela E: Platelet-rich-fibrin: A novel root coverage approach. J Indian Soc Periodontol 13: 50-54, 2009.
21. He L, Lin Y, Hu X, Zhang Y and Wu H: A comparative study of platelet-rich fibrin (PRF) and platelet-rich plasma (PRP) on the effect of proliferation and differentiation of rat osteoblasts in vitro. Oral Surg Oral Med Oral Pathol Oral Radiol Endod 108: 707-713, 2009.

22. Dohan DM, Choukroun J, Diss A, Dohan SL, Dohan AJ, Mouhyi J and Gogly B: Platelet-rich fibrin (PRF): A second-generation platelet concentrate. Part III: Leucocyte activation: A new feature for platelet concentrates? Oral Surg Oral Med Oral Pathol Ora Radiol Endod 101: e51-e55, 2006.

23. Naik B, Karunakar P, Jayadev M and Marshal VR: Role of Platelet rich fibrin in wound healing: A critical review. J Conserv Dent 16: 284-293, 2013.

24. Pripatnanont $P$, Nuntanaranont $T$, Vongvatcharanon $S$ and Phurisat K: The primacy of platelet-rich fibrin on bone regeneration of various grafts in rabbit's calvarial defects. J Craniomaxillofac Surg 41: e191-e200, 2013.

25. Xuan F, Lee CU, Son JS, Jeong SM and Choi BH: A comparative study of the regenerative effect of sinus bone grafting with platelet-rich fibrin-mixed Bio-Oss ${ }^{\circledR}$ and commercial fibrin-mixed Bio-Oss ${ }^{\circledR}$ : An experimental study. J Craniomaxillofac Surg 42: e47-e50, 2014.

26. Hoaglin DR and Lines GK: Prevention of localized osteitis in mandibular third-molar sites using platelet-rich fibrin. Int J Dent 2013: 875380, 2013.

27. Peck MT, Marnewick J and Stephen L: Alveolar ridge preservation using leukocyte and platelet-rich fibrin: A report of a case. Case Rep Dent 2011: 345048, 2011.

28. Barone A, Ricci M, Romanos GE, Tonelli P, Alfonsi F and Covani U: Buccal bone deficiency in fresh extraction sockets: A prospective single cohort study. Clin Oral Implants Res 26: 823-830, 2015 .

29. Hauser F, Gaydarov N, Badoud I, Vazquez L, Bernard JP and Ammann P: Clinical and histological evaluation of postextraction platelet-rich fibrin socket filling: A prospective randomized controlled study. Implant Dent 22: 295-303, 2013.

30. Singh A, Kohli M and Gupta N: Platelet rich fibrin: A novel approach for osseous regeneration. J Maxillofac Oral Surg 11: 430-434, 2012

31. Dohan DM, Choukroun J, Diss A, Dohan SL, Dohan AJ, Mouhyi J and Gogly B: Platelet-rich fibrin (PRF): A second-generation platelet concentrate. Part I: Technological concepts and evolution. Oral Surg Oral Med Oral Pathol Oral Radiol Endod 101: e37-e44, 2006

32. Fickl S, Zuhr O, Wachtel H, Bolz W and Huerzeler M: Tissue alterations after tooth extraction with and without surgical trauma: A volumetric study in the beagle dog. J Clin Periodontol 35: 356-363, 2008

33. Beck TM and Mealey BL: Histologic analysis of healing after tooth extraction with ridge preservation using mineralized human bone allograft. J Periodontol 81: 1765-1772, 2010.

34. Cypher TJ and Grossman JP: Biological principles of bone graft healing. J Foot Ankle Surg 35: 413-417, 1996.

35. Eriksson C, Ohlson K, Richter K, Billerdahl N, Johansson M and Nygren $\mathrm{H}$ : Callus formation and remodeling at titanium implants. J Biomed Mater Res A 83: 1062-1069, 2007.

36. Suttapreyasri S and Leepong N: Influence of Platelet-rich fibrin on alveolar ridge preservation. J Craniofac Surg 24: 1088-1094, 2013.

37. Schropp L, Wenzel A, Kostopoulos L and Karring T: Bone healing and soft tissue contour changes following single-tooth extraction: A clinical and radiographic 12-month prospective study. Int J Periodontics Restorative Dent 23: 313-323, 2003.

38. Araujo MG and Lindhe J: Dimensional ridge alterations following tooth extraction. An experimental study in the dog. J Clin Periodontol 32: 212-218, 2005.

39. Fickl S, Zuhr O, Wachtel H, Stappert CF, Stein JM and Hürzeler MB: Dimensional changes of the alveolar ridge contour after different socket preservation techniques. J Clin Periodontol 35: 906-913, 2008

40. Choukroun J, Diss A, Simonpieri A, Girard MO, Schoeffler C, Dohan SL, Dohan AJ, Mouhyi J and Dohan DM: Platelet-rich fibrin (PRF): A second-generation platelet concentrate. Part IV: Clinical effects on tissue healing. Oral Surg Oral Med Oral Pathol Oral Radiol Endod 101: e56-e60, 2006.

This work is licensed under a Creative Commons Attribution-NonCommercial-NoDerivatives 4.0 International (CC BY-NC-ND 4.0) License. 